\title{
Coupled Simulation of Seismic Wave Propagation and Failure Phenomena by Use of an MPS Method
}

\author{
Junichi Takekawa, ${ }^{1}$ Hitoshi Mikada, ${ }^{2}$ Tada-nori Goto, ${ }^{3}$ Yoshinori Sanada, ${ }^{4}$ and Yuzuru AshidA ${ }^{5}$
}

\begin{abstract}
The failure of brittle materials, for example glasses and rock masses, is commonly observed to be discontinuous. It is, however, difficult to simulate these phenomena by use of conventional numerical simulation methods, for example the finite difference method or the finite element method, because of the presence of computational grids or elements artificially introduced before the simulation. It is, therefore, important for research on such discontinuous failures in science and engineering to analyze the phenomena seamlessly. This study deals with the coupled simulation of elastic wave propagation and failure phenomena by use of a moving particle semi-implicit (MPS) method. It is simple to model the objects of analysis because no grid or lattice structure is necessary. In addition, lack of a grid or lattice structure makes it simple to simulate large deformations and failure phenomena at the same time. We first compare analytical and MPS solutions by use of Lamb's problem with different offset distances, material properties, and source frequencies. Our results show that analytical and numerical seismograms are in good agreement with each other for 20 particles in a minimum wavelength. Finally, we focus our attention on the Hopkinson effect as an example of failure induced by elastic wave propagation. In the application of the MPS, the algorithm is basically the same as in the previous calculation except for the introduction of a failure criterion. The failure criterion applied in this study is that particle connectivity must be disconnected when the distance between the particles exceeds a failure threshold. We applied the developed algorithm to a suspended specimen that was modeled as a long bar consisting of thousands of particles. A compressional wave in the bar is generated by an abrupt pressure change on one edge. The
\end{abstract}

1 Kyoto University, C1-1-111, Kyotodaigaku-Katsura, Nishikyo-ku, Kyoto 615-8540, Japan. E-mail: takekawa@tansa. kumst.kyoto-u.ac.jp

2 Kyoto University, C1-1-112, Kyotodaigaku-Katsura, Nishikyo-ku, Kyoto 615-8540, Japan. E-mail: mikada@gakushikai.jp

3 Kyoto University, C1-1-113, Kyotodaigaku-Katsura, Nishikyo-ku, Kyoto 615-8540, Japan. E-mail: goto.tadanori.8a@ kyoto-u.ac.jp

4 Japan Agency for Marine-Earth Science and Technology (JAMSTEC), 3173-25, Showa-machi, Kanazawa-ku, Yokohama, Kanagawa 236-0001, Japan. E-mail: sanada@jamstec.go.jp

5 Environment, Energy, Forestry and Agriculture Network (EEFA), Suzakuhaitsu4-G, 27-1, Mibusuzaku-cho, Nakakyo-ku, Kyoto 604-8871, Japan. E-mail: ashida@eefa.jp compressional wave propagates along the interior of the specimen and is visualized clearly. At the other end of the bar, the spalling of the bar is reproduced numerically, and a broken piece of the bar is formed and falls away from the main body of the bar. Consequently, these results show that the MPS method effectively reproduces wave propagation and failure phenomena at the same time.

Key words: Moving particle semi-implicit method, discontinuous phenomenon, failure, elastic wave propagation.

\section{Introduction}

Several numerical methods have been developed and modified for modeling elastic wave propagation. Finite difference methods (FDM) with the staggered grid technique (VIrIEUX, 1986; Graves, 1996) have been widely used to solve elastic wave equations because of the simplicity and accuracy of the methods. Finite element methods (FEM) and spectral element methods (SEM) have also been used, because of their flexibility in respect of geometric topography (KoKETSu et al., 2004; КоматітSCH and Tromp, 1999). These methods use grids or elements structures to discretize the object.

Mesh-free methods which need no connectivity between nodes and elements have also been developed (Nayroles et al., 1992; Belytschko et al., 1994). These were applied to elastic wave propagation modeling (JiA and Hu, 2006; Katou et al., 2009). Although these methods provide accurate simulation results in elastic wave propagation problems, implementation of failure phenomena related to wave propagation has not been considered in depth. A discrete element method (DEM), one of the particle methods, was developed to deal with granular materials (Cundall and Strack, 1979), and applied to 
failure simulations (BRARA et al., 2001; POTYONDY and Cundall, 2004). DEM has also been applied to elastic wave propagation. Del Valle-Garcia and Sanchez-Sesma (2003) and O'Brien and Bean (2004) simulated surface wave propagation and evaluated the accuracy of their methods. O'BRIEN et al. (2009) evaluated the dispersion property of an elastic lattice method and showed the applicability of the method to seismic modeling in a complex Earth model. These studies on discrete or particle methods focused mainly on seismic wave propagation.

In this study, we apply a moving particle semiimplicit method (MPS) to failure phenomena and elastic wave propagation at the same time. The MPS method was developed to analyze incompressible fluid flow. Dam-break problems and breaking wave analysis are applications of the MPS method (KosHIZUKA and OKA, 1996; KoshizUKa et al., 1998, 1999a). The method has also been applied to solid analysis and fluid-structure interaction analysis (KoshizuKA et al., 1999b; Chikazawa et al., 2001a, b). Because the method can handle large deformations or fragmentation of solids, use of the simulation to deal with the coupling between elastic wave propagation and failure phenomena can be achieved seamlessly.

In this paper, we first compare analytical and MPS solutions by use of Lamb's problem with different offset distances, material properties, and source frequencies. We then simulate the Hopkinson effect as an example of failure phenomena induced by elastic wave propagation.

\section{Methods}

\subsection{Particle Interaction Model}

In the MPS method the elastic body is represented as an assembly of particles. Each particle interacts with neighboring particles and a weighting function $\mathrm{w}(r)$ is used to calculate the differential operators in the governing equations.

$$
\mathrm{w}(r)=r_{\mathrm{e}} / r-1\left(r \leq r_{\mathrm{e}}\right), 0\left(r>r_{\mathrm{e}}\right)
$$

where $r$ is the distance between particles and $r_{\mathrm{e}}$ is the radius of the influence domain. Each particle interacts solely with particles inside the influence domain. Particle number density $n_{i}$ is defined as:

$$
n_{i}=\sum_{j \neq \mathrm{i}} \mathrm{w}(r)
$$

Calculated particle density number will be constant if the particle arrangement is uniform. This constant value is denoted by $n^{0} \cdot n^{0}$ is used as a normalizing factor when the particle interactions are averaged by use of the weighting function.

In the MPS method, differential operators are modeled as the interactions between particles. For example, gradient and divergence are modeled as follows:

$$
\begin{aligned}
\langle\nabla \phi\rangle_{i} & =\frac{d}{n^{0}} \sum_{j \neq \mathrm{i}} \frac{\left(\phi_{j}-\phi_{i}\right)\left(r_{j}-r_{i}\right)}{\left|r_{j}-r_{i}\right|^{2}} \mathrm{w}\left(\left|r_{j}-r_{\mathrm{i}}\right|\right) \\
\langle\nabla \cdot \mathbf{u}\rangle_{i} & =\frac{2 d}{n^{0}} \sum_{i \neq j} \frac{\left(\mathbf{u}_{j}-\mathbf{u}_{i}\right)\left(r_{j}-r_{i}\right)}{\left|\mathrm{r}_{j}-\mathrm{r}_{i}\right|^{2}} \mathrm{w}\left(\left|\mathbf{r}_{j}-\mathbf{r}_{i}\right|\right)
\end{aligned}
$$

where $\phi$ and $\mathbf{u}$ are arbitrary variables, $d$ is the number of space dimensions, and $\mathbf{r}_{i}$ and $\mathbf{r}_{j}$ are the positioning vectors of particles $i$ and $j$. You can find the more details of particle interaction models in KoshizuKA and OKA (1996).

\subsection{Governing Equation for Elastic Body}

In this section, we explain the MPS method algorithm for elastic body analysis. In the MPS method, both an explicit scheme (KoshizuKa et al., 1999b) and an implicit scheme (ChiKAzAwa et al., 2001a) exist. Here, we adopt the explicit scheme to simulate elastic wave propagation and dynamic fracturing. Although we focus on two-dimensional problems for simplicity in this study, the following concept could readily be applied to three-dimensional (3D) problems except for calculation of the rotational angle. Because the rotational angle is not scalar but a vector in $3 \mathrm{D}$, calculation of the rotational angle is slightly complex. The increment of numerical costs (CPU time and memory) largely depends on the influence domain, i.e. the number of neighboring particles. When we use the radius of the influence domain $r_{\mathrm{e}}=1.9 \times \Delta x(\Delta x$ is the particle spacing in a regular lattice), the number of particles in 2D and 3D cases are 8 and 26, respectively. This means the computational cost in 3D becomes more than triple that in 2D. Therefore, the influence domain needs to be smaller for efficient calculations 
without reducing the accuracy. The effects of the influence domain on accuracy are investigated by use of Lamb's problem in the next section.

The governing equation for an elastic body is as follows:

$$
\rho \partial v_{\alpha} / \partial t=\partial / \partial x_{\beta}\left(\lambda \varepsilon_{\gamma \gamma} \delta_{\alpha \beta}+2 \mu \varepsilon_{\alpha \beta}\right)+\rho f_{\alpha}
$$

where $\mathbf{v}_{\alpha}$ is the velocity vector, $\rho$ is the mass density, $\lambda$ and $\mu$ are Lame's constants, $\varepsilon_{\alpha \beta}$ is the strain tensor, $\delta_{\alpha \beta}$ is Kronecker delta, and $\mathbf{f}_{\alpha}$ is the external force vector. The strain tensor can be described as follows:

$$
\varepsilon_{\alpha \beta}=\left(\partial \mathrm{u}_{\alpha} / \partial x_{\beta}+\partial \mathrm{u}_{\beta} / \partial \mathrm{x}_{\alpha}\right) / 2
$$

where $\mathbf{u}_{\alpha}$ is the displacement vector.

Each particle has position, velocity, rotational angle, and angular velocity of degrees of freedom. The relative displacement vector between particles $i$ and $j$ can be described as follows:

$$
\begin{gathered}
\mathbf{u}_{i j}=\mathbf{r}_{i j}-R \mathbf{r}_{i j}^{0} \\
\mathbf{r}_{i j}=\mathbf{r}_{j}-\mathbf{r}_{i} \\
\mathbf{r}_{i j}^{0}=\mathbf{r}_{j}^{0}-\mathbf{r}_{i}^{0} \\
R=\left(\begin{array}{cc}
\cos \theta_{i j} & -\sin \theta_{i j} \\
\sin \theta_{i j} & \cos \theta_{i j}
\end{array}\right)
\end{gathered}
$$

where $\mathbf{u}_{i j}$ is the relative displacement vector between particles $i$ and $j, R$ is the rotation matrix, $\mathbf{r}_{i j}$ and $\mathbf{r}_{i j}^{0}$ are the relative position vectors of the present and initial conditions, respectively. $\theta_{i j}$ is the averaged rotational angle between particles $i$ and $j$. In this way, the relative displacement between particles is calculated by eliminating the rotational component.

In the MPS method, the stress and strain between particles is calculated as a vector as follows:

$$
\begin{aligned}
& \boldsymbol{\sigma}_{i j}^{n}=2 \mu \varepsilon_{i j}^{n}=2 \mu \mathbf{u}_{i j}^{n} /\left|\mathbf{r}_{i j}^{0}\right| \\
& \boldsymbol{\sigma}_{\mathrm{ij}}^{\mathrm{s}}=2 \mu \varepsilon_{\mathrm{ij}}^{\mathrm{s}}=2 \mu \mathbf{u}_{\mathrm{ij}}^{\mathrm{s}} /\left|\mathbf{r}_{\mathrm{ij}}^{0}\right|
\end{aligned}
$$

where $\mathbf{u}_{i j}^{n}$ and $\mathbf{u}_{i j}^{s}$ are the relative displacement vector between particles $i$ and $j$ in the normal and tangential components of the $\mathbf{r}_{i j}$ direction, respectively. $\boldsymbol{\sigma}_{i j}^{n}$ and $\boldsymbol{\sigma}_{i j}^{s}$ are the stress components in normal and tangential directions, respectively. All components of the stress tensor are not needed to calculate the acceleration of particles. $\varepsilon_{\gamma \gamma}$ in Eq. (5) corresponds to the volumetric strain term and is calculated by use of the following equations.

$$
\begin{aligned}
&\left(\varepsilon_{\gamma \gamma}\right)_{i}=(\operatorname{div} \mathbf{u})_{i} \\
&= \frac{d}{n^{0}} \sum_{i \neq j} \frac{\mathbf{u}_{i j} \cdot \mathbf{r}_{i j}}{\left|\mathrm{r}_{i j}^{0}\right|\left|\mathrm{r}_{i j}\right|} \mathrm{w}\left(\left|\mathbf{r}_{i j}^{0}\right|\right) \\
&= \frac{d}{n^{0}} \sum_{i \neq j} \varepsilon_{i j}^{n} \frac{\mathrm{r}_{i j}}{\left|\mathrm{r}_{i j}\right|} \mathrm{w}\left(\left|\mathbf{r}_{i j}^{0}\right|\right) \\
& p_{i}=-\lambda\left(\varepsilon_{\gamma \gamma}\right)_{i}
\end{aligned}
$$

where $p_{i}$ is the pressure on particle $i$. We can calculate the acceleration of particles by using stresses and pressure $\left(\boldsymbol{\sigma}_{i j}^{n}, \boldsymbol{\sigma}_{i j}^{n}, p_{i}\right)$.

$$
\begin{array}{r}
{\left[\frac{\partial \mathbf{v}_{i}}{\partial t}\right]=\left[\frac{\partial \mathbf{v}_{i}}{\partial t}\right]_{n}+\left[\frac{\partial \mathbf{v}_{i}}{\partial t}\right]_{\mathrm{s}}+\left[\frac{\partial \mathbf{v}_{i}}{\partial t}\right]_{\mathrm{p}}} \\
\rho_{i}\left[\frac{\partial \mathbf{v}_{i}}{\partial t}\right]_{n}=\frac{\mathrm{d}}{n^{0}} \sum_{i \neq j} \frac{2 \sigma_{i j}^{n}}{\left|\mathbf{r}_{i j}^{0}\right|} \mathrm{w}\left(\left|\mathbf{r}_{i j}^{0}\right|\right) \\
\rho 1_{i}\left[\frac{\partial \mathbf{v}_{i}}{\partial t}\right]_{\mathrm{s}}=\frac{d}{n^{0}} \sum_{i \neq j} \frac{2 \sigma_{i j}^{s}}{\left|\mathbf{r}_{i j}^{0}\right|} \mathrm{w}\left(\left|\mathbf{r}_{i j}^{0}\right|\right) \\
\rho_{i}\left[\frac{\partial \mathbf{v}_{i}}{\partial t}\right]_{\mathrm{p}}=-\frac{d}{n^{0}} \sum_{i \neq j} \frac{2 p_{i j} \mathbf{r}_{i j}}{\left|\mathbf{r}_{i j}^{0}\right|\left|\mathbf{r}_{i j}\right|} \mathrm{w}\left(\left|\mathbf{r}_{i j}^{0}\right|\right)
\end{array}
$$

where $p_{i j}$ is the pressure between particles $i$ and $j$ calculated as follows:

$$
p_{i j}=\left(p_{i}+p_{j}\right) / 2
$$

In Eqs. (15)-(18), we used the divergence model in Eq. (4).

In this method, for conservation of angular momentum, rotations are added to particles to cancel the torque generated by tangential stress. The force acting on particle $i$ as a result of tangential stress is calculated by use of the equation:

$$
\mathbf{F}_{i j}=m_{i}\left[\frac{\partial \mathbf{v}_{i j}}{\partial t}\right]_{\mathrm{s}}=\frac{\mathrm{m}_{i}}{\rho_{i}} \frac{2 d}{n^{0}} \frac{\sigma_{i j}^{s}}{\left|\mathbf{r}_{i j}^{0}\right|} \mathrm{w}\left(\left|\mathbf{r}_{i j}^{0}\right|\right)
$$

where $m_{i}$ is the mass of particle $i$. On the other hand, particle $j$ is subjected to an opposite force which has the same absolute value. The torque $\mathbf{T}_{i j}$ generated by a couple of force given by Eq. (20) is also calculated as follows: 


$$
\mathbf{T}_{i j}=-\mathbf{r}_{i j} \times \mathbf{F}_{i j}
$$

The torque calculated by use of Eq. (21) is added to particles $i$ and $j$.

$$
I_{i}\left[\frac{\partial \omega_{i}}{\partial t}\right]=I_{j}\left[\frac{\partial \omega_{j}}{\partial t}\right]=-\frac{1}{2} T_{i j}
$$

where $I_{i}$ is the moment of inertia of particle $i$.

From the equations given above, we can update the velocity, position, angular velocity, and rotational angle of particle $i$ as follows:

$$
\begin{gathered}
\mathbf{v}_{i}^{k+1}=v_{\mathrm{i}}^{\mathrm{k}}+\Delta t\left[\partial \mathbf{v}_{i} / \partial t\right]^{k} \\
\mathbf{r}_{i}^{k+1}=\mathbf{r}_{i}^{k}+\Delta t \mathbf{v}_{i}^{k+1} \\
\omega_{i}^{k+1}=\omega_{i}^{k}+\Delta t\left[\partial \omega_{i} / \partial t\right]^{k} \\
\theta_{\mathrm{i}}^{\mathrm{k}+1}=\theta_{\mathrm{i}}^{\mathrm{k}}+\Delta \mathrm{t} \omega_{\mathrm{i}}^{\mathrm{k}+1}
\end{gathered}
$$

If we consider the two-dimensional problem, the equation for the rotational angle becomes a scalar expression. This scheme is symplectic. The accuracy of the scheme described above is verified in the next section by use of surface wave propagation.

\section{Numerical Simulation of Surface Wave Propagation}

In this section, we verify the reproducibility of the surface wave field in the MPS method by comparison with an analytical solution of Lamb's problem. We studied two models (A and B) which are homogeneous and isotropic. Model $\mathrm{A}$ has a P-wave velocity of $V_{\mathrm{p}}=2,611 \mathrm{~m} / \mathrm{s}$, an $S$-wave velocity of $V_{\mathrm{S}}=$ $1,846 \mathrm{~m} / \mathrm{s}$, and a mass density of $\rho=2,200 \mathrm{~kg} / \mathrm{m}^{3}$. Model B has a P-wave velocity of $V_{\mathrm{p}}=4,522 \mathrm{~m} / \mathrm{s}$, an S-wave velocity of $V_{\mathrm{S}}=1,846 \mathrm{~m} / \mathrm{s}$, and a mass density of $\rho=2,200 \mathrm{~kg} / \mathrm{m}^{3}$. The particle distance $\Delta x$ and time spacing $\Delta t$ are $0.1 \mathrm{~m}$ and $0.01 \mathrm{~ms}$, respectively. The time spacing is set to satisfy the Courant condition. The simulations are conducted for two different radii of the influence domain (i.e. $1.9 \times \Delta x$ and $2.1 \times \Delta x$ ) for comparison. The number of particles inside the influence domain is 8 and 12 in each case. The compressional seismic source and eight receivers are set at $1 \mathrm{~m}$ depth. The distance between the seismic source and the nearest receiver is $10 \mathrm{~m}$, and the spacing of each receiver is $4 \mathrm{~m}$. The source function used in the simulation is the first derivative of a Gaussian function with central frequencies of 300 and $400 \mathrm{~Hz}$.

We compute the misfit between analytical and numerical results to evaluate the effect of offset distance, material property, radius of the influence domain, and source frequency. The misfit is calculated by use of the equation:

$$
\text { Misfit }=\frac{\sum_{t}\left(S^{\mathrm{NUM}}(t)-S^{\mathrm{ANA}}(t)\right)^{2}}{\sum_{t} S^{\mathrm{ANA}}(t)^{2}}
$$

where $S^{\mathrm{NUM}}(t)$ is the seismogram from the MPS method and $S^{\mathrm{ANA}}(t)$ is the analytical seismogram.

Figures 1 and 2 show the seismograms and misfits recorded at the receivers for different source frequencies. The influence domain is set to $1.9 \times \Delta x$ in each figure. Solid and dashed lines are analytical and numerical results, respectively. The dotted lines are the difference between them amplified by a factor of 5. The misfits calculated by use of Eq. (27) increase with an increase in the offset distances in each case. The vertical direction has larger misfits than the horizontal in both medium properties and frequencies.

The misfits of vertical seismograms for model $\mathrm{B}$ are smaller than those for model $\mathrm{A}$, because the velocity of the Rayleigh wave for model B is slightly higher than that for model A. On the other hand, the misfits of horizontal seismograms for model B are slightly larger than those for model A, especially in the far receivers. This is caused by the small amplitude of far receivers in model $\mathrm{B}$, which induces the small value of the denominator in Eq. (27).

On the other hand, the difference relating to the source frequency is clear; i.e. the misfits for $300 \mathrm{~Hz}$ are smaller than those for $400 \mathrm{~Hz}$. This is caused by the change in the number of particles in a wavelength. The number of particles in a minimum wavelength for 300 and $400 \mathrm{~Hz}$ are approximately 20 and 15 , respectively. It is well known that a small number of grids or particles in a wavelength leads to numerical dispersion. These results show that the misfit in the farthest receiver, whose offset distance is approximately 20 wavelengths, can be limited to less than $10 \%$ if we use approximately 20 particles in a minimum wavelength. 
(a)

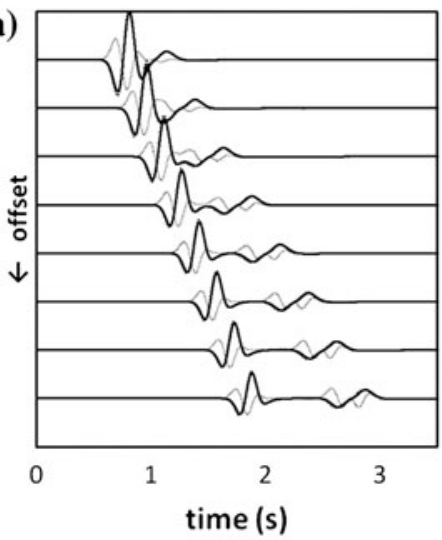

(b)

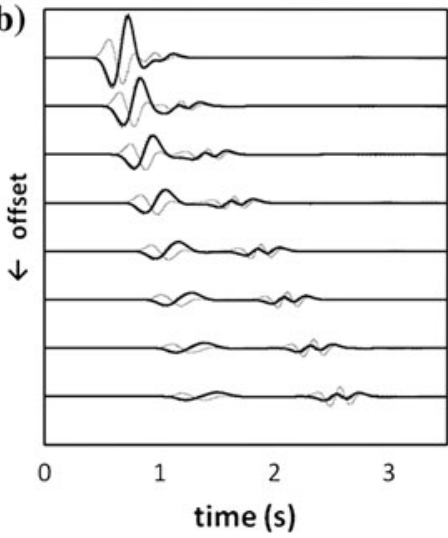

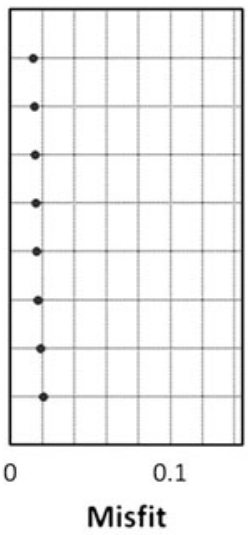

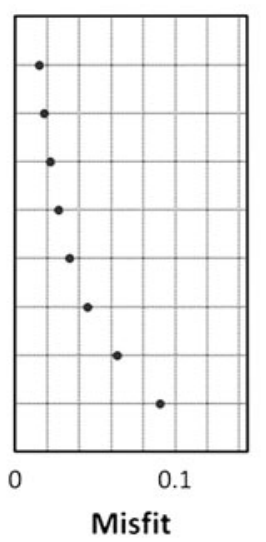

(c)

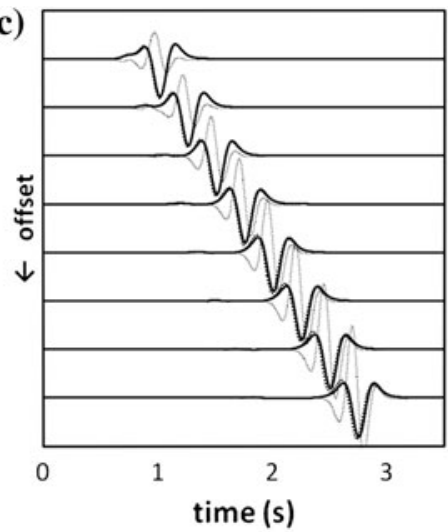

(d)

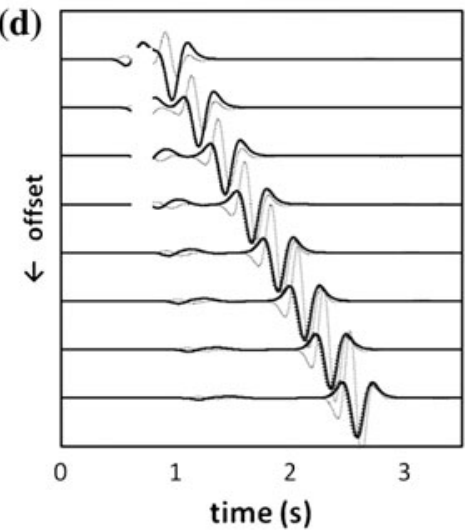

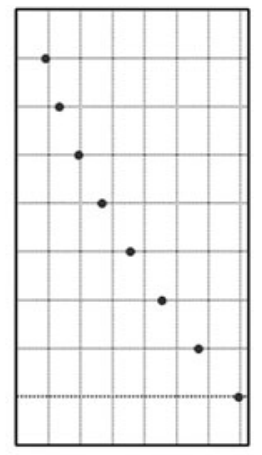

0.1

Misfit

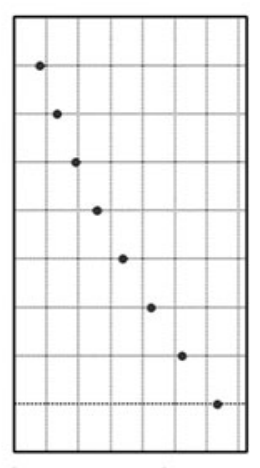

0

0.1

Misfit

Figure 1

Comparison of numerical and analytical seismograms. The radius of the influence domain is set to $1.9 \times \Delta x$. The source frequency is $400 \mathrm{~Hz}$. Solid, dashed, and dotted lines are analytical, numerical, and differences between them, respectively. Filled circles are misfits of each seismogram calculated by use of Eq. (27). a Horizontal displacement at the receivers for model A. b Horizontal displacement at the receivers for model B. c Vertical displacement at the receivers for model A. d Vertical displacement at the receivers for model B

Figure 3 shows the seismograms and misfits recorded at the receivers for an influence domain of $2.1 \times \Delta x$. The source frequency is set to $400 \mathrm{~Hz}$. It is observed that the difference of the misfits is indistinguishable from that in Fig. 1. This indicates that the smaller influence domain has an advantage in terms of calculation cost (CPU time and memory) that would be saved by using the smaller number of neighboring particles.

\section{Numerical Simulation of Dynamic Tensile Fracturing}

We conducted numerical simulations of Hopkinson's effect as an example of failure phenomenon induced by elastic wave propagation. Figure 4 shows a schematic diagram of the experimental condition of the split Hopkinson pressure bar. The incident compressive wave generated at an end of Hopkinson bar is transmitted into the specimen, and is reflected at the other free end of the specimen as a tensile wave. Because of the superposition of the incident compressive wave and the reflected tensile wave, the tensile stress which leads to spalling of the specimen is generated near the free end. This phenomenon has been used to determine the dynamic tensile strength of brittle material such as a rock mass. Many researchers have conducted numerical simulations of Hopkinson's effect by use of diverse methods (BRARA et al., 2001; Сно et al., 2003; ZHU and TANG, 2006).

Introduction of a failure criterion is a key aspect of this study. Brara et al., (2001) adopted the cumulative fracture criterion proposed by Klepaczko 
(a)

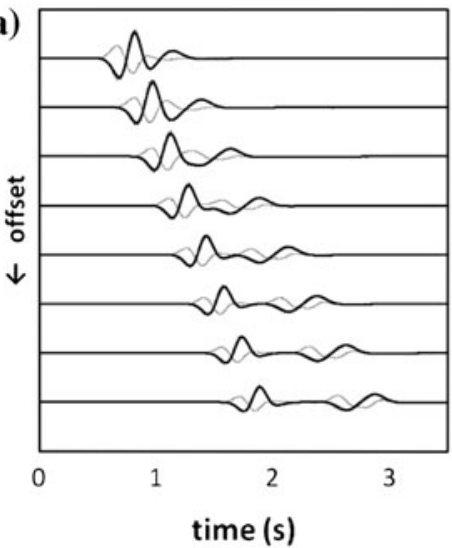

(b)

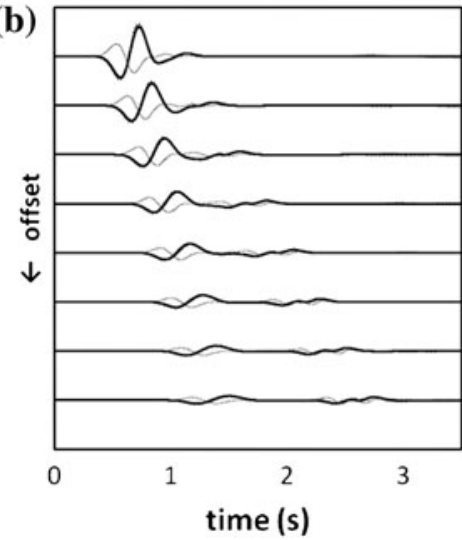

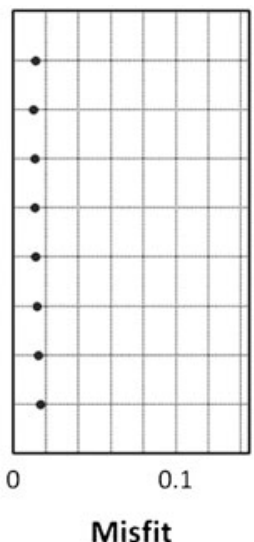

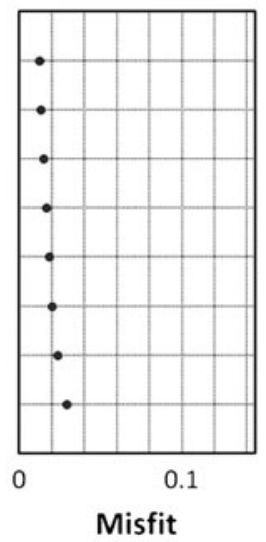

(c)

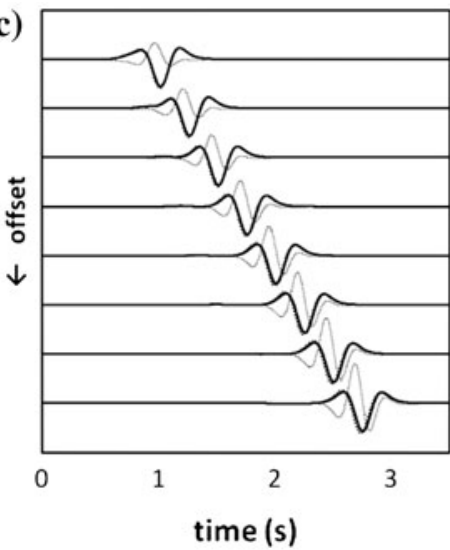

(d)

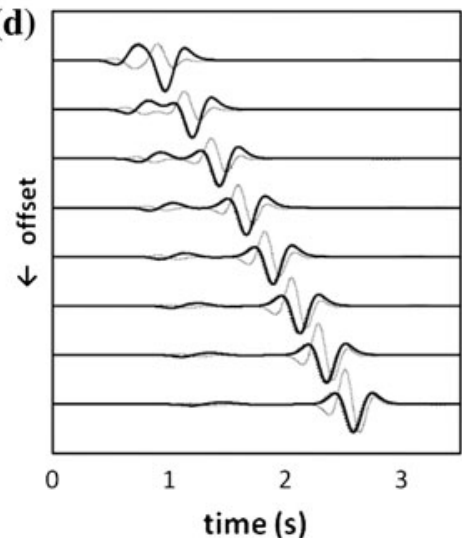

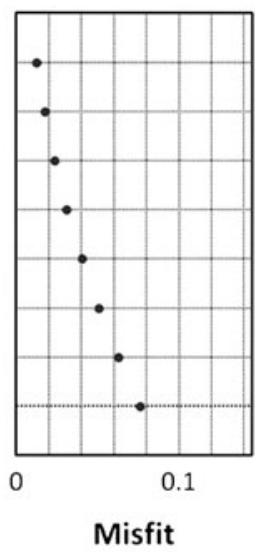

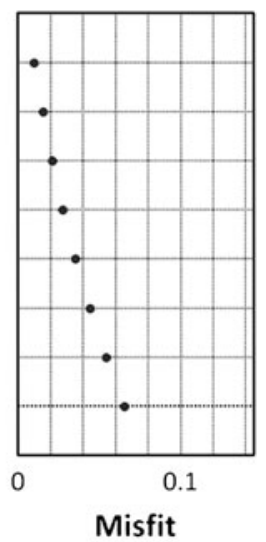

Figure 2

Comparison of numerical and analytical seismograms. The radius of the influence domain is set to $1.9 \times \Delta x$. The source frequency is $300 \mathrm{~Hz}$. Details are given in caption of Fig. 1. a Horizontal displacement at the receivers for model A. b Horizontal displacement at the receivers for model B. c Vertical displacement at the receivers for model A. d Vertical displacement at the receivers for model B

(1990). ZHU and TANG (2006) used the rate-dependent failure criterion proposed by Zнао (2000). In this study we adopted a very simple failure criterion. If the distance between particles exceeds a threshold value, the interaction between particles is set to zero. This failure criterion is very simple to implement although only tensile failure can be reproduced. In the simulation of Hopkinson's effect, this failure criterion is sufficient because only tensile failure will be generated in the bar.

We discretize the split Hopkinson pressure bar by using MPS particles. The width and the height of the bar are $300 \mathrm{~mm}$ and $10 \mathrm{~mm}$, respectively. The model is discretized into $301 \times 11$ particles. The particle distance and the time spacing are $1 \mathrm{~mm}$ and $0.0001 \mathrm{~ms}$, respectively. Three models, in which homogeneous and isotropic media are assumed, are studied. Model A has a P-wave velocity of $V_{\mathrm{p}}=$ $2,993 \mathrm{~m} / \mathrm{s}$, an S-wave velocity of $V_{\mathrm{S}}=1,600 \mathrm{~m} / \mathrm{s}$, and a mass density of $\rho=2,000 \mathrm{~kg} / \mathrm{m}^{3}$. Model B has a P-wave velocity of $V_{\mathrm{p}}=2,613 \mathrm{~m} / \mathrm{s}$, an $\mathrm{S}$-wave velocity of $V_{\mathrm{S}}=1,600 \mathrm{~m} / \mathrm{s}$, and a mass density of $\rho=2,000 \mathrm{~kg} / \mathrm{m}^{3}$. Model $\mathrm{C}$ has a P-wave velocity of $V_{\mathrm{p}}=2,400 \mathrm{~m} / \mathrm{s}$, an S-wave velocity of $V_{\mathrm{S}}=$ $1,600 \mathrm{~m} / \mathrm{s}$, and a mass density of $\rho=2,000 \mathrm{~kg} / \mathrm{m}^{3}$. We apply the impulsive force to one edge to generate the compressive wave in the bar. In this study, we use the time history of a Gaussian function.

Figure 5 shows the pressure and velocity distributions in the bar near the right edge. The impulsive force is applied at the left end of the bar. After approximately $0.16 \mathrm{~ms}$, the compressive pressure 
(a)

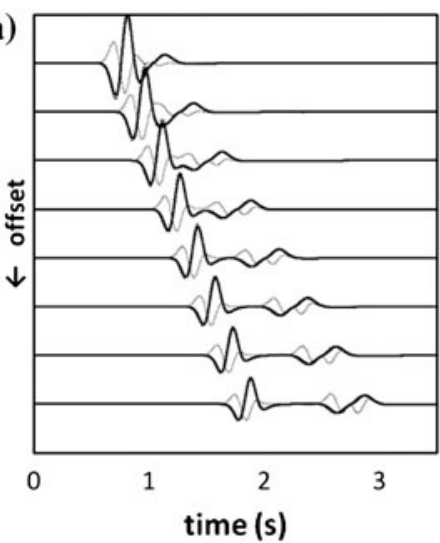

(b)

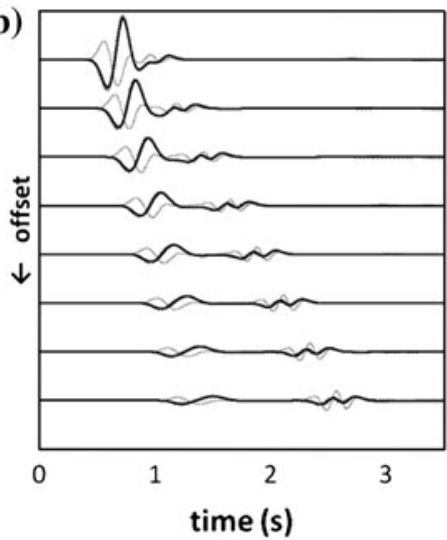

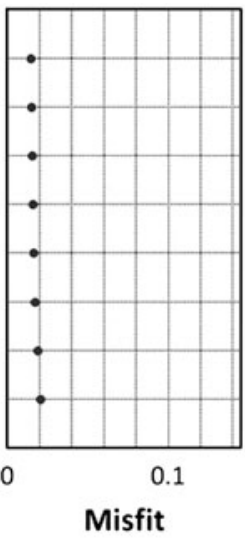

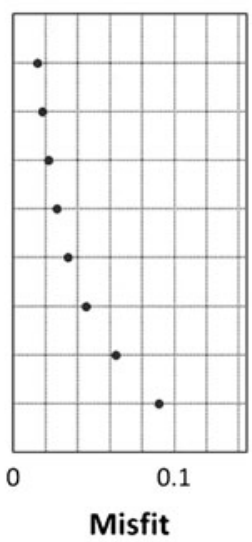

(c)

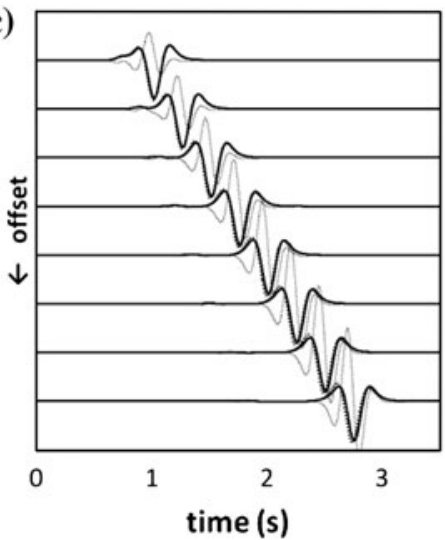

(d)

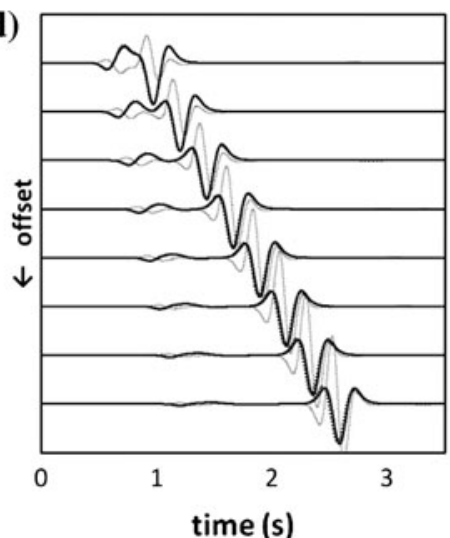

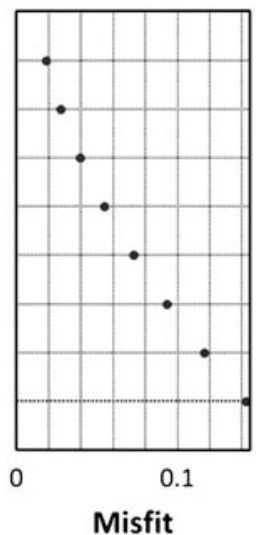

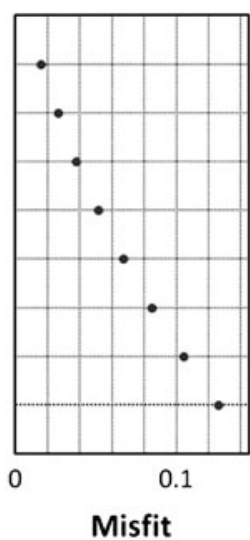

Figure 3

Comparison of numerical and analytical seismograms. The radius of the influence domain is set to $2.1 \times \Delta x$. The source frequency is $400 \mathrm{~Hz}$. Details are given in caption of Fig. 1. a Horizontal displacement at the receivers for model A. b Horizontal displacement at the receivers for model B. c Vertical displacement at the receivers for model A. d Vertical displacement at the receivers for model B

wave reaches the other edge of the bar. Because of the free boundary condition, the reflected pressure wave is generated with tensile stress. At $0.17 \mathrm{~ms}$, the tensile pressure wave propagating in the opposite direction is observed. After $0.18 \mathrm{~ms}$, the discontinuity of the velocity field indicated by an arrow becomes visible because of the detachment of neighboring particles. The fragmented block flies away to rightward with local oscillation.

Figure 6 shows the particle distribution only around the right edge in several time steps. At $0.180 \mathrm{~ms}$, particles start to separate near the free end in each model. At $0.200 \mathrm{~ms}$, we can observe the clear spalling of the piece of the bar. In each model, dynamic fracturing induced by elastic wave propagation can be reproduced in a similar manner. However, the lengths of the fragments in both models are different. The longest and shortest pieces are observed in models A and C, respectively. This is caused by the different transmissive wavelengths because of the different P-wave velocity. In model A, the transmissive wavelength is longer than in the other models. On the other hand, model $\mathrm{C}$ has the shortest wavelength. The difference of wavelength changes the distance between the location where the maximum tensile stress is generated and the free end. Therefore, the length of the fragment in model $\mathrm{A}$ is longer than those of the other models.

Here, we compare fracture cross-sections from our numerical results with those from previous numerical and experimental results. In BRARA et al. (2001), the fracture cross-section simulated by DEM did not have a flat surface, unlike our numerical results. Additionally, the results from Сно et al. 
Impulsive force is applied to one edge

Impulsive force is applied to one edge
brittle material

Compressive wave propagates.

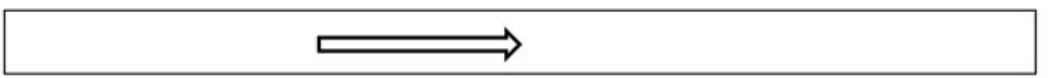

Compressive wave is converted to tensile wave.

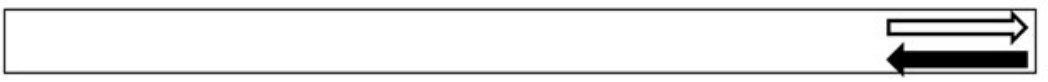

Dynamic spalling is generated at another edge.

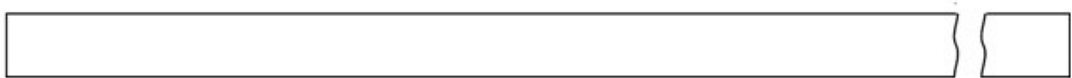

Figure 4

Schematic diagram of Hopkinson's effect

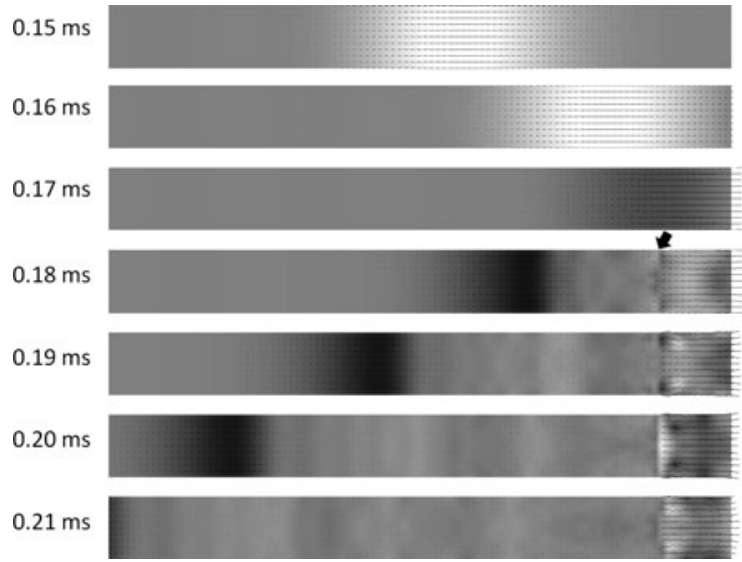

Figure 5

Pressure and velocity distributions in the bar near the right edge. White and black color represent compressional and tensile pressure. Solid lines represent velocity vectors

(2003) also have a similar non-flat fracture surface. BRARA et al. (2001) used random packing of particles for their DEM simulations; the heterogeneous property is included in their numerical model. This leads to a non-linear fracture, because failure will occur selectively depending on the alignment of particles. Сно et al. (2003) used uniform triangular elements for their finite element analysis. However, the spatial distribution of local strength satisfying Weibull's distribution was used to consider the inhomogeneous property of rock. Thus, selective local failures, which cause a non-flat fracture cross-section, were also induced by the distribution of strength criterion. Because natural rock is an inhomogeneous material, and the inhomogeneity has a significant effect on the shape of the fracture cross-section, the fracture surface is indented according to their numerical results. On the other hand, our numerical model does not include the inhomogeneous property; i.e. regular lattice structure and constant failure criterion are used. This is the reason for the different shape of the fracture cross-section in our results and previous results. If we introduce an inhomogeneous property, the non-flat failure surface will be reproduced, although it exceeds the purpose of this study.

It is difficult to compare the length of fragment of our results with those from other numerical or experimental results because the experimental conditions described above are different from each other. In our results, however, the tensile fragmentations occur near the end, as in previous numerical and experimental studies (BRARA et al., 2001; CHO et al., 2003). This therefore indicates the MPS method can 
(a)

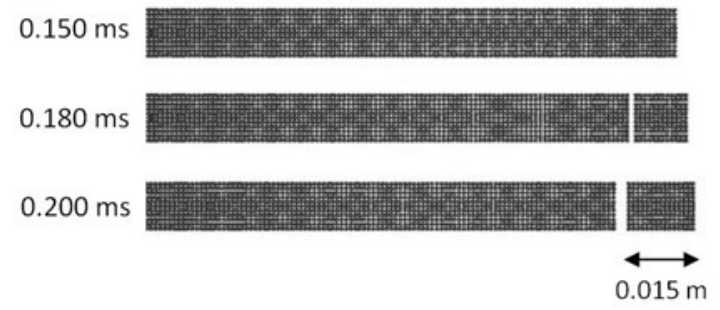

(b)

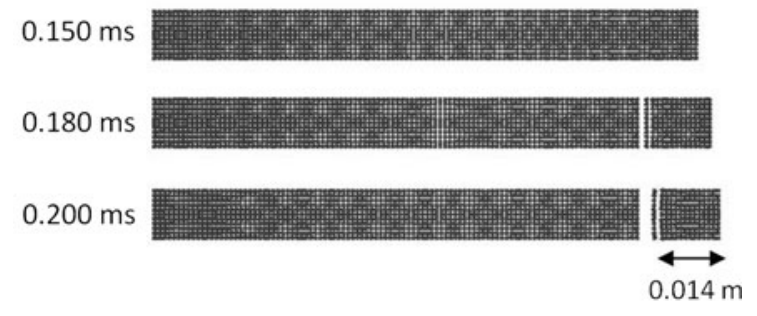

(c)

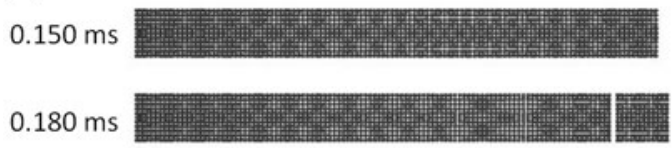

$0.200 \mathrm{~ms}$

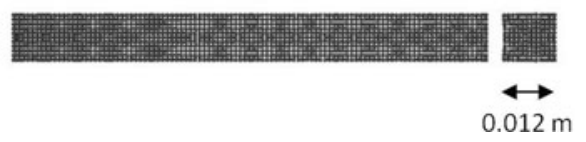

Figure 6

a The particle configuration in three time steps for model A. b The particle configuration in three time steps for model B. c The particle configuration in three time steps for model C

seamlessly reproduce both elastic wave propagation and dynamic fracturing.

\section{Conclusions}

In this study, we conducted numerical simulations of elastic wave propagation and failure phenomenon using an MPS method.

First, we verified the dispersion properties of the MPS method by comparing numerical and analytical solutions using Lamb's problem. We changed offset distances, medium properties, and source frequencies, and evaluated the misfit of each condition. The results showed that the misfit can be smaller than $10 \%$ if we use 20 particles per minimum wavelength for a propagation of approximately 20 wavelengths.
We then reproduced Hopkinson's effect, as an example of failure phenomena induced by elastic wave propagation, by use of the MPS method. In the simulation, material failure is represented by setting the interaction between particles to zero. We studied three numerical models with different material properties. The results of numerical experiments agree with previous results from experimental or other numerical methods. This indicates that dynamic fracturing induced by elastic wave propagation can be reproduced by the MPS method.

\section{Acknowledgments}

This study was supported by MEXT/JSPS KAKENHI Grant Number 24760361. We thank anonymous reviewers for their thoughtful comments and suggestions.

\section{REFERENCES}

Alford, R. M., Kelly, K. R., and Boore, D. M. (1974), Accuracy of finite-difference modeling of the acoustic wave equation, Geophysics, 39, 834-842.

Belytschko, T., Lu, Y. Y., and Gu, L. (1994), Element-free Galerkin methods, Int. J. Num. Meth. Eng., 37, 229-256.

Brara, A., Camborde, F., Klepaczko, J. R., and Mariotti, C. (2001), Experimental and numerical study of concrete at high strain rates in tension, Mech. Mater., 33, 33-45.

Cerjan, C., Kosloff, D., Kosloff, R., and Reshef, M. (1985), A nonreflecting boundary condition for discrete acoustic and elastic wave equations, Geophysics, 50, 705-708.

Chikazawa, Y., Koshizuka, S., and Ока, Y. (2001a), A particle method for elastic and visco-plastic structures and fluid-structure interactions, Compt. Mech., 27, 97-106.

Chikazawa, Y., Koshizuka, S., and OKa, Y. (2001b), Numerical analysis of three-dimensional Sloshing in an Elastic Cylindrical Tank using Moving Particle Semi-implicit Method, Comput. Fluid Dynamics J., 9, 376-383.

Cho., S. H., Ogata, Y., and Kaneko, K. (2003), Strain-rate dependency of the dynamic tensile strength of rock, Int. J. Rock Mech. Min. Sci., 40, 763-777.

Cundall, P. A., and Strack, O. D. L. (1979), A discrete numerical model for granular assemblies, Geotechnique, 29, 47-65.

Del Valle-Garcia, R., Sanchez-Sesma, F. J. (2003), Rayleigh waves modeling using an elastic lattice model, Geophys. Res. Lett., 30, 1866.

Graves, R. W. (1996), Simulating seismic wave propagation in $3 D$ elastic media using staggered-grid finite differences, Bull. Seism. Soc. Am., 86, 1091-1106.

JiA, X., and Hu, T. (2006) Element-free precise integration method and its applications in seismic modeling and imaging, Geophys. J. Int., 166, 349-372. 
Katou, M., Matsuoka, T., Mikada, H., Sanada, Y., and Ashida, Y. (2009), Decomposed element-free Galerkin method compared with finite-difference method for elastic wave propagation, Geophysics, 74, H13-H25.

KLEPACZKo, J. R. (1990), Behavior of rock like materials at high strain rates in compression, Int. J. Plasticity, 6, 415-432.

Koketsu, K., Fujiwara, H., and Ikegami, Y. (2004), Finite-element simulation of seismic ground motion with a voxel mesh, Pure appl. Geophys., 161, 2183-2198.

Komatiтsch, D., and Tromp, J. (1999), Introduction to the spectral element method for three-dimensional seismic wave propagation, Geophys. J. Int., 139, 806-822.

Koshizuka, S., and Ока, Y. (1996), Moving particle semi-implicit method for fragmentation of incompressible fluid, Nucl. Sci. Eng., 123, 421-434.

KoshizuKa, S., Noвe, A., and Ока, Y. (1998), Numerical analysis of breaking waves using the moving particle semi-implicit method, Int. J. Num. Meth. Fluids, 26, 751-769.

Koshizuka, S., IKeda, H., and Ока, Y. (1999a), Numerical analysis of fragmentation mechanisms in vapor explosions, Nucl. Eng. Des., 189, 423-433.

Koshizuka, S., Chikazawa, Y., Ока, Y. (1999b), Development of an explicit particle calculation model for elastic structures, Proceeding of the Conference on Computational Engineering and Science, 33-36. (in Japanese).
Nayroles, B., Touzot, G., and Villon, P. (1992), Generalizing the finite element method : diffuse approximation and diffuse elements, Comput. Mech., 10, 307-318.

O'Brien, G. S., BEAN, C. J. (2004), A 3D discrete numerical elastic lattice method for seismic wave propagation in heterogeneous media with topography, Geophys. Res. Lett., 31, L14608.

O'Brien, G. S., Bean, C. J., and Tapamo, H. (2009), Dispersion analysis and computational efficiency of elastic lattice methods for seismic wave propagation, Comput. Geosci., 35, 1768-1775.

Park, D., Jeon, B., and Jeon, S. (2009), A numerical study of the screening of blast-induced waves for reducing ground vibration, Rock Mech. Rock Eng., 42, 449-473.

Potyondy, D. O., and Cundall, P. A. (2004), A bonded-particle model for rock, Int. J. Rock Mech. Min. Sci., 41, 1329-1364.

ToOmey, A., and BeAn, C. (2000), Numerical simulation of seismic waves using a discrete particle scheme, Geophys. J. Int., 141, 595-604.

Virieux, J. (1986), P-SV wave propagation in heterogeneous media : Velocity-stress finite difference method, Geophysics, 51, 889-901.

Zнао, J. (2000), Application of Mohr-Coulomb and Hoek-Brown strength criteria to the dynamic strength of brittle rock, Int. J. Rock Mech. Min. Sci., 37, 105-112.

Zhu, W. C., and TANG, C. A. (2006), Numerical simulation of Brazilian disk rock failure under static and dynamic loading, Int. J. Rock Mech. Min. Sci., 43, 236-252. 